\title{
Implants in Glaucoma-An Overview
}

\author{
Dr. Bhawesh chandra saha ${ }^{1,}$ Dr.Rashmi kumari ${ }^{2}$, Dr.Atul anand ${ }^{3,}$ \\ Dr.Santosh kumar ${ }^{4}$ Dr.B P Sinha ${ }^{5}$ \\ ${ }^{1,3}$ Senior Resident,AIIMS,Patna,${ }_{5}{ }_{5}$ Senior resident ,IGIMS ,Patna ${ }^{4}$ junior Resident,IGIMS,Patna \\ ${ }^{5}$ Professor,HOD,IGIMS ,Patna \\ All India Institute Of Medical Sciences(AIIMS),Patna Indira Gandhi Institute Of Medical \\ Sciences,(IGIMS),Patna
}

\begin{abstract}
Glaucoma is a multifactorial optic neuropathy in which there is a characteristic acquired loss of retinal ganglion cell and atrophy of optic nerve. Glaucoma filteration surgery (GFS) has been shown to be more effective at preventing disease progression than other primary treatment modalities in glaucoma. Glaucoma drainage devices or implants are being used more frequently in the treatment of glaucoma that does not respond to medications or trabeculectomy operations.They too have undergone evolution in the past decade and this review intends to compile the infornation along with recent modifications and future perspective after a thorough literature search.
\end{abstract}

Keywords: Glaucoma,drainage devices,implants

\section{Introduction}

Glaucoma is a multifactorial optic neuropathy in which there is a characteristic acquired loss of retinal ganglion cell and atrophy of optic nerve. ${ }^{1}$.Although the etiology of glaucomatous optic neuropathy is multifactorial, elevated IOP is a major risk factor and lowering intraocular pressure still remains the mainstay of treatment modalities of glaucoma.

Glaucoma filtration surgery (GFS) has been shown to be more effective at preventing disease progression than other primary treatment modalities in glaucoma. ${ }^{3}$ Glaucoma drainage devices are being used more frequently in the treatment of glaucoma that does not respond to medications or trabeculectomy operations. Glaucoma drainage devices (GDDs) create an alternate aqueous pathway from the anterior chamber by channeling aqueous out of the eye through a tube to a subconjunctival bleb or to the suprachoroidal space. ${ }^{4,5}$ This tube is usually connected to an equatorial plate under the conjunctiva A variety of materials have been used to facilitate aqueous drainage from the anterior chamber, including silk thread, glass, platinum, teflon, cartilage, and autologous lacrimal canaliculus. ${ }^{8-12}$. These early implants were associated with high complication rates, excessive scar formation near the limbus, seton migration and conjunctival erosion. Molteno introduced the implant consisting of a long silicon tube attached to a large end plate placed 9-10 $\mathrm{mm}$ posterior to the limbus. The modern implants are based on this concept of Molteno. ${ }^{17}$

Currently, the GDDs are divided into three categories;

- GDD with no resistance

- GDD with resistance and

- GDD with variable resistance to aqueous outflow.

\section{GDDs with no resistance;}

These implants consist of a silicon tube connected to an end plate placed sub conjunctively which acts as surface for bleb formation. The Molteno implants are of two types: single plate and double plate. A single plate Molteno implant is a silicon tube with external diameter of $0.63 \mathrm{~mm}$ and internal diameter of $0.30 \mathrm{~mm}$ connected to the upper surface of a polypropylene plate ${ }^{18}$. The double plate implant consists of two plates one of which is attached to silicon tube in the anterior chamber while the second connects the two plates forming a surface area of $270 \mathrm{~mm} 2$.The Baerveldt implant consists of a silicon tube attached to a barium impregnated silicon plate with a surface area of $200 \mathrm{~mm}^{2}, 350 \mathrm{~mm}^{2}$ or $500 \mathrm{~mm}^{2}$. The advantage of the Baerveldt implant is that it has a large surface area plate that can be implanted through a one-quadrant conjunctival incision ${ }^{19}$

The Schocket implant is a cheap and easily assembled implant which includes a silastic tube used for NLD intubation. This tube is connected to a $360^{\circ}$ encircling silicon band, like that used in retinal detachment surgery. The disadvantage of this implant is that the surgical procedure of implantation is cumbersome and lengthy. 


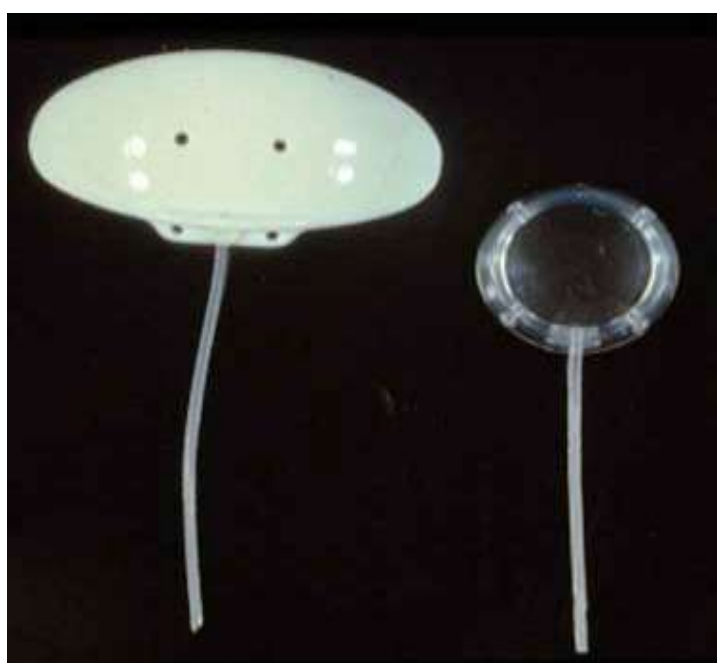

Fig;- Baerveldt and Molteno implant (single plate)

\section{GDDs with set resistance;}

To avoid early post operative complications such as excessive drainage and hypotony, the concept of a one-way valve that opens at predetermined IOP was introduced by Krupin and associates .The Ahmed Glaucoma Valve; AGV consists of a silicon tube attached to a silicon sheet valve held in a polypropylene body. ${ }^{20}$ The valve consists of a thin silicon elastomer membrane which creates a venturi-shaped chamber. Because the inlet is wider than the outlet, a pressure gradient between the anterior chamber and the bleb is created which enables the valve to open in response to pressure differential, as described by Bernouillis principle. The valve is designed to open at an IOP of $8 \mathrm{~mm}$ of $\mathrm{Hg}$ or more. The Optimed implant is made up of a silicon tube with PMMA plate. The flow is restricted by the presence of an element in the rectangular box situated at the end of the tube within the plate. The Ahmed glaucoma valve (AGV) has lowest incidence of hypotony amongst all valved devices.

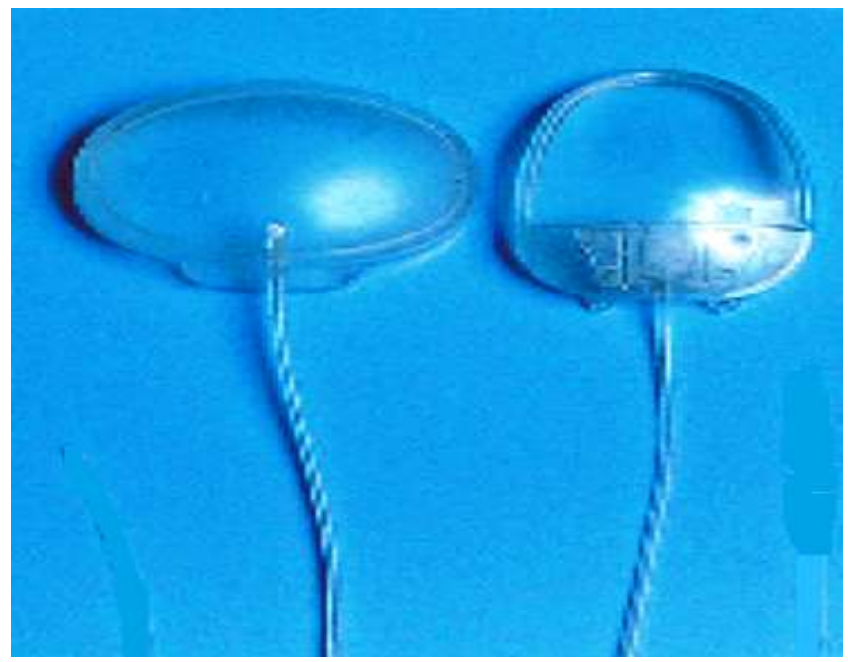

Fig;- Krupin slit valve and Ahmed glaucoma valve

\section{GDDs with variable resistance;}

These are modified Molteno and Baerveldt implants. These devices include a tissue resistance mechanism that limits the aqueous flow. However, since the tissue apposition force is variable, IOP levels remain unpredictable. The Molteno implant with a pressure ridge is a dual chamber, single plate implant with a $\mathrm{V}$-shaped pressure ridge on the upper surface of the plate which encloses an area of $10.5 \mathrm{~mm}^{2}$ around the opening of silicon tube. The pressure ridge and the overlying Tenon's capsule regulate the flow of aqueous into the main bleb cavity during the early post-operative period, thereby decreasing excessive filtration and hypotony. But these complications are not effectively prevented by the pressure ridge mechanism.

The Baerveldt bioseal is a flap that overhangs the silicon tube as it opens on the end plate. The apposition of bioseal elements to the sclera with absorbable sutures provides early resistance, which limits the aqueous flow beneath the device. 


\section{Pathophysiology;}

After implantation of the GDD, there is formation of a fibrous capsule around the end plate over a period of several weeks. The fibroblasts do not adhere to the silicone or polypropylene material of the plate. This is an important feature which allows success of drainage implant. The aqueous humor passes out of the anterior chamber and collects in the space between the end plate and non-adherent fibrous capsule. Aqueous flow then passes through the fibrous capsule via passive diffusion and is absorbed by periocular lymphatics.

The fibrous capsule is the main site of resistance to aqueous outflow. Therefore, the success of drainage surgery is dependent on capsular thickness and surface area of encapsulation. The thinner is the capsule and larger the surface area of encapsulation, the lower will be the intraocular pressure. A large plate will have an increased surface area of encapsulation and greater intra-ocular pressure (IOP) reduction. Heuer and colleagues achieved a higher success rate and a better IOP reduction with the double plate implant due to its increased surface area

Indications; Glaucoma drainage devices are associated with serious intraoperative and post operative complications, hence these are reserved for patients with refractory and intractable glaucoma.

GDD devices are indicated for

- Neovascular glaucoma,

- Glaucoma with uveitis,

- Refractory infantile glaucoma,

- Failed filtration surgery,

- Patients with ocular surface diseases like pemphigus, and

- Secondary glaucoma following penetrating keratoplasty, retinal detachment and cataract surgery.

Contraindications; GDDs are associated with various postoperative complications and hence are contraindicated in noncompliant patients. They are not recommended for patients with poor endothelial function.

\section{Surgical technique;}

The surgical technique is the same for most implants. A fornix based conjunctival flap is made in the superotemporal or superonasal quadrant with two relaxing incisions. The superotemporal quadrant is preferred because a supero-nasal approach may induce postoperative strabismus (Prata et al 1993). A partial thickness rectangular scleral flap is created as large as possible so that the tube is covered. The draining part of the implant is placed in the sub-tenon's space and the anterior portion is sutured to the sclera approximately $7 \mathrm{~mm}$ posterior to the limbus.The silicon tube is radially placed across scleral flap and excess tube is trimmed so as to overlap the limbus by $2 \mathrm{~mm}$. A 23 gauge needle is used to enter the anterior chamber and the tube is inserted through this opening. The scleral flap is sutured with 10-0 nylon. The conjunctiva is attached back around the limbus.

Due to larger dimensions, Baerveldt and Schocket implants require dissection in one or more than one quadrant. A scleral patch graft from donor tissue may be placed over the tube to avoid postoperative erosion of tube as a modification of original technique .

\section{Post-operative period}

Hypotensive phase; After surgery this phase lasts from day 1 to 4 weeks. During this phase the bleb looks to be diffuse and thick valved with few engorged blood vessels. The IOP is low and varies from 2-3 mm of $\mathrm{Hg}$ to $10-$ $12 \mathrm{~mm}$ of $\mathrm{Hg}$.

Hypertensive phase; Beginning 3-6 weeks after surgery, it can last for 4- 6 months. The bleb appears inflamed; dome shaped and may be associated with a raised IOP of $30 \mathrm{mmHg}$ or greater. The Ahmed glaucoma valve has increased incidence of hypertensive phase when compared to Baerveldt implant and the double plate Molteno as shown in many studies. The increased incidence can be explained because of increased surface area of these two implants. The difference may also be due to the different bio-materials used in various implants.

Stable phase; After the hypertensive phase there is stabilization of IOP in the mid to high teens. The bleb appears as a thick-valved, dome-shaped, elevated area overlying the end plate with no associated inflammation.

\section{Intraoperative complications;}

\section{Complications}

Globe perforation and uveal tissue exposure, vitreous loss, can occur while fixing the implant.

Intra-operative complications can be hyphema, supra-choroidal hemorrhage or expulsion, vitreous hemorrhage, ciliary body bleeding while inserting the tube.

If the incision is large there can be leakage around the implant. 


\section{Post operative complications; ${ }^{24}$}

One of the major post-operative complications of GDD is shallow anterior chamber secondary to wound leak, overfiltration, and choroidal effusion. The incidence of over-filtration is higher in nonvalved implants. To address this complication, ligatures can be placed around the external portion of the tube or the internal lumen can be occluded. An 8-0 suture can be tied around intraocular portion of the tube and cut after one week with an argon laser. Hypotony from overfiltration usually can be left as such unless there is a flat anterior chamber with lens-cornea touch. In this case, the anterior chamber should be reformed with a viscoelastic agent. In recalcitrant cases, one needs to repeat the procedure. Choroidal effusions can be managed conservatively with steroids orally or topically. Large effusions require surgical drainage. Raised intraocular pressure (IOP) may be encountered in the early post operative period, and can be due to fibrin, vitreous, an iris plug occluding the lumen, or a tight external ligature. The techniques which have been reported to open the occlusion include irrigation of the tube with saline through paracentesis or use of Nd-YAG Laser for vitreous incarceration. A tight suture can be severed with an argon laser. A late increase in intraocular pressure can be due to a thick fibrous capsule. and can also be the result of topical steroids used during the postoperative period (Mermoud \& Salmon, 1993).

Ocular motility disturbances may occur following large plate implant, which manifest as diplopia and strabismus. When implanted in inferonasal quadrants, larger plates can interrupt extra ocular movements. It can be corrected by a replacement with a smaller design, transfer to another quadrants or in persistent cases, removal of the implant. The intraocular portion of the tube may touch the cornea leading to corneal edema and decompensation. The tube-cornea touch can be minimised by using a scleral patch graft instead of a scleral flap. Alternatively, the tube can be repositioned (Freedman,1987). Retraction of the tube may occur as a result of inadequate anchoring of the tube to episclera. Late erosion of tube may also occur. Epithelial ingrowth is uncommon, but may occur in tubes inserted closer to limbus.

Other late complications include sterile hypopyon, choroidal effusion, choroidal hemorrhage, retinal detachment, endophthalmitis and phthsis bulbi. Endophthalmitis as a result of drainage device implantation is not very common; the incidence is less than 2\% (Al Torbak et al 2005). Early postoperative endophthalmitis may be associated with host flora while late onset endophthalmitis is more common in thin-walled and leaky blebs that allow trans-conjuctival migration of bacteria (Geddeet al 2001).

Due to the high risk of complications, the US Food and Drug Administration has classified these devices as Category 3, presenting a potential unreasonable risk requiring the highest level of regulation.

\section{Recent advances in GDDs;}

Recently, many advances have been made in the field of GDDs with respect to materials, design, and technique of implantation. All implants share the common goal of shunting aqueous humor out of the anterior chamber and bypassing the trabecular meshwork to increase outflow and lower the IOP.

Some recently introduced implants are described below.

Ex-Press shunt (Stainless steel) ${ }^{\mathbf{2 3 , 2 4}}$; The Express shunt is the latest development in the treatment of difficult glaucoma cases. It consists of a $3 \mathrm{~mm}$ long stainless steel tube with a central hollow lumen that is 400 micrometers in external diameter and 50 micrometers internally. The bleb formation starts immediately and micro cysts within the bleb can often be seen within the first or second post operative day. Additionally, surgery with the Express is less time consuming than with a larger shunt and, if it fails, a more extensive shunt procedure can be planned later.

The Ex-Press shunt was initially designed to implant near the limbus through sclera into the anterior chamber. The external plate of the shunt was placed under the conjunctival flap thereby producing a filtering bleb near the entrance of Ex- Press shunt. This led to a number of postoperative complications including hypotony, choroidal detachment and suprachoroidal hemorrhage.To overcome these complications, the Ex-Press shunt is implanted under the scleral flap which reduces the overall complication rate. ${ }^{25,26}$ 


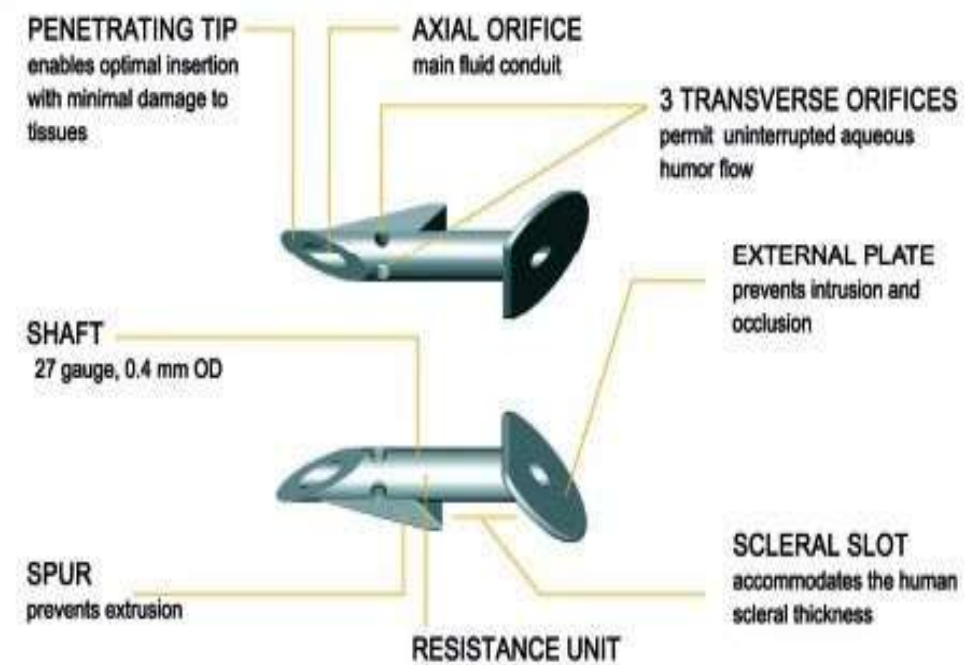

Fig: Ex-Press shunt

Gold micro shunt (GMS); This device uses a 24 carat gold plate which is implanted into the suprachoroidal space. It is a biocompatible and inert material. The IOP reduction is achieved by the presence of opening and closing holes in the gold microshunt. The outflow is titrated with the help of $790 \mathrm{~nm}$ Titanium sapphire laser.

Artificial nano drainage implant (ANDI); ANDIs are a biomedical device with a characteristic design that increases its success rate. It is a serpentine microchannel made of poly dimethyl siloxane (PDMS). The microchannel regulates the forward flow by friction. The special serpentine design increases the length of channel thereby creating a larger pressure differential over a smaller area. It also decreases the chances of infection by impending bacteria movement up the device .

Glaukos iStent; It is a Titanium device which is placed inside the Schlemm's canal, thereby allowing the aqueous humor to flow directly into the canal and bypassing the trabecular meshwork. The advantage of this implant is that it is free from bleb related complications. It is implanted through a clear corneal incision under topical anaesthesia.

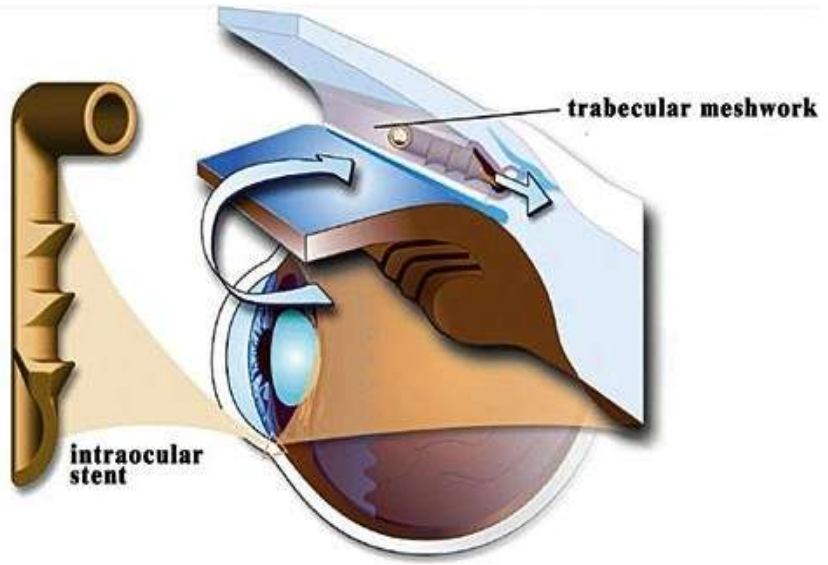

Fig; Glaukos iStent

Eyepass glaucoma implant; It is a Y-shaped stent made of silicon. The Eyepass directly shunts aqueous humor from the anterior chamber into the Schemm's canal bypassing the trabecular meshwork. The arms of the Yshaped implant facilitate the flow of aqueous in both the clockwise and counter clockwise direction. The rate of complications is also reduced. More studies and long term follow up is required to determine the safety and utility of this implant and any additional advantage over the currently used GDDs.

Aquaflow glaucoma implant; A non-penetrating deep sclerectomy along with the aqua flow collagen glaucoma implant has shown significantly lower post operative complications and better outcomes when compared to conventional trabeculectomy . 
The Aquaflow glaucoma implant (ologen) is a $5 \mathrm{~mm} \times 4 \mathrm{~mm}$ in length. The collagen implant is inserted under the scleral flap after a deep sclerectomy. It swells to double its original size after absorption of eye fluids, and the implant takes 6 to 9 months to dissolve. The normal time for surgical scar healing is less than the life of this device. Once the aqua flow is dissolved, a channel remains to allow the aqueous flow to exit from the desired location, thereby maintaining the reduced IOP. Since the surgeon does not enter the anterior chamber, the chances of cataract formation are also reduced.

\section{Future perspective;}

Currently, an aqueous shunt is in clinical trials which will include the formation of a thinner capsule and have greater hydraulic conductivity. It is based on the hypothesis that changing the geometrical design of the commonly used shunt devices from a plate design to a cylindrical shape will reduce the tension on the capsule surrounding the implant.The goals of new designs are to produce easier to use implants with fewer complications, and more predictable IOP control. Currently, none of the available implants provide predictable resistance to fluid outflow.

\section{Conclusion}

Glaucoma drainage devices have been successfully used to treat glaucoma in refractory cases but due to high rates of complications, they are reserved for cases of recalcitrant glaucoma. In developing countries with illiteracy and poor follow-up trabeculectomy with Mitomycin-C remains the treatment of choice.

\section{References}

[1]. Van Buskirk EM, Cioffi GA. Glaucomatous optic neuropathy. Am J Ophthalmol. 1992 Apr 15. 113(4):447-52.

[2]. Bathija R, Gupta N, Zangwill L, Weinreb RN. Changing definition of glaucoma. J Glaucoma. 1998 Jun. 7(3):165-9.

[3]. Jay JL, Allan D. The benefit of early trabeculectomy versus conventional management in primary open angle glaucoma relative to the severity of the disease. Eye 1989;3:528-35.

[4]. Hill RA, Nguyen QH, Baerveldt G, et al. Trabeculectomy and Molteno implantation for glaucomas associated with uveitis. Ophthalmology 1993;100:903-908.

[5]. Sidoti PA, Dunphy TR, Baerveldt G, et al. Experience with the Baerveldt glaucoma implant in treating neovascular glaucoma. Ophthalmology 1995;102:1107-1118.

[6]. Da Mata A, Burk SE, Netland PA, et al. Management of uveitic glaucoma with Ahmed glaucoma valve implantation. Opthalmology 1999; 106:2168-2172.

[7]. Ceballos EM, Parrish RK, Schiffman JC. Outcome of Baerveldt glaucoma drainage implants for the treatment of uveitic glaucoma. Ophthalmology 2002;109:2256-2260.G

[8]. Ayyala RS, Harman LE, Michelini-Norris B, et al. Comparison of different biomaterials for glaucoma drainage devices. Arch Ophthalmol 1999;117:233-236.

[9]. Ayyala RS, Michelini-Norris B, Flores A, et al. Comparison of different biomaterials for glaucoma drainage devices: part 2. Arch Ophthalmol 2000;118;1081-1084.

[10]. Mackenzie PJ, Schertzer RM, Isbister CM. Comparison of silicone and polypropylene Ahmed glaucoma valves: two-year followup. Can J Ophthalmol 2007;42:227-232.

[11]. Brasil MVOM, Rockwood EJ, Smith S. Comparison of silicone and polypropylene Ahmed glaucoma valve implants. J Glaucoma 2007;16:36-41.

[12]. Ishida K, Netland PA, Costa VP, et al. Comparison of polypropylene and silicone Ahmed glaucoma valves. Ophthalmology 2006;113:1320-1326.

[13]. Sarkisian SR, Netland PA. Tube extender for revision of glaucoma drainage implants. J Glaucoma2007;16:637-639.

[14]. Tessler Z, Jluchoded S, Rosenthal G. Nd:YAG laser for Ahmed tube shunt occlusion by the posterior capsule. Ophthlamic Surg Lasers 1997;28:69-70.

[15]. Netland PA, Schuman S. Management of glaucoma drainage implant tube kink and obstruction with pars plana clip. Ophthalmic Surg Lasers Imaging 2005;36:167-168.

[16]. Tarbak AAA, Shahwan SA, Jadaan IA, et al. Endophthalmitis associated with the Ahmed glaucoma valve implant. Br J Ophthalmol 2005;89:454-458.

[17]. Munoz M, Parrish RK. Prospective evaluation of extraocular motility following double-plate Molteno implantation. Arch Ophthalmol1999;117;1155-1160.

[18]. Freedman J. Clinical Experience with the Molteno dual-chamber single-plate implant. OphthalmicSurg 1992;23:238-241.

[19]. Syed HM, Law SK, Nam SH, et al. Baerveldt-350 implant versus Ahmed valve for refractory glaucoma: a case-controlled comparison. J Glaucoma 2004;13:38-45.

[20]. Wang JC, See JL, Chew PT. Experience with the use of Baerveldt and Ahmed glaucoma drainage implants in an Asian population. Ophthalmology 2004;111:1383-1388.

[21]. Djodeyre MR, Calvo JP, Gomez JA. Clinical evaluation and risk factors of time to failure of Ahmed glaucoma valve implant in pediatric patients. Ophthalmology 2001;108:614-620.

[22]. Wilson MR, Mendis U, Smith SD, et al. Ahmed glaucoma valve implant vs. trabeculectomy in the surgical treatment of glaucoma: A randomized clinical trial. Am J Ophthalmol 2000;130:267-273.

[23]. Wilson MR, Mendis U, Paliwal A, et al. Long-term follow-up of primary glaucoma surgery with Ahmed glaucoma valve implant versus trabeculectomy. Am J Ophthalmol 2003;136:464-470.

[24]. Kanner EM Netland PA Sarkisian SR Du H . Ex-PRESS miniature glaucoma device implanted under a scleral flap alone or combined with phacoemulsification cataract surgery. J Glaucoma. 2009;18:488-491.

[25]. Bissig A Feusier M Mermoud A Roy S . Deep sclerectomy with the Ex-PRESS X-200 implant for the surgical treatment of glaucoma. Int Ophthalmol. 2010;30(6):661-668.

[26]. de Feo F Bagnis A Bricola G Scotto R Traverso CE. The efficacy and safety of a steel drainage device implanted under a scleral flap. Can J Ophthalmol. 2009;44:457-462. 